\title{
A Short Review on Cancer Biomarkers
}

\author{
Nalini Kanta Sahoo*, Madhusmita Sahu \\ MLR Institute of Pharmacy, Dundigal, Medchal, Telangana, Hyderabad
}

*Corresponding Author: Nalini Kanta Sahoo, MLR Institute of Pharmacy, Dundigal, Medchal, Telangana, Hyderabad, Email: sahoo.nalini@gmail.com

\begin{abstract}
A biomarker may be a molecule secreted by a tumor or a specific response of the body to the presence of cancer. Genetic, epigenetic, proteomic, glycomic, and imaging biomarkers can be used for cancer diagnosis, prognosis, and epidemiology. Biomarkers have a lot of applications in oncology, which includes risk assessment, screening, differential diagnosis, determination of prognosis, prediction of response to treatment, and monitoring of progression of disease. Because of major role that bio-markers play at all stages of disease, it is mandatory that they should undergo rigorous evaluation, including analytical validation, clinical validation, and assessment of clinical utility, prior to incorporation into routine clinical care. In this review we discuss about the development of biomarkers, including types and various applications.
\end{abstract}

Keywords: Epigenetic, proteomics, Prognosis, biomarkers

\section{INTRODUCTION}

A cancer biomarker refers to a substance or process that is indicative of the presence of cancer in the body. A biomarker may be a molecule secreted by a tumor or a specific response of the body to the presence of cancer. Genetic, epigenetic, proteomic, glycomic, and imaging biomarkers can be used for cancer diagnosis, prognosis, and epidemiology. Ideally, such biomarkers can be assayed in noninvasively collected biofluids like blood or serum.

While numerous challenges exist in translating biomarker research into the clinical space; a number of gene and protein based biomarkers have already been used at some point in patient care; including, AFP (Liver Cancer), BCR-ABL (Chronic Myeloid Leukemic), BRCA1 / BRCA2 (Breast/Ovarian Cancer), BRAF V600E (Melanoma/Colorectal Cancer), CA-125 (Ovarian Cancer), CA19.9 (Pancreatic Cancer), CEA (Colorectal Cancer), EGFR (Non-smallcell lung carcinoma), HER-2 (Breast Cancer), KIT (Gastrointestinal stromal tumor), PSA (Prostate Specific Antigen) (Prostate Cancer), S100 (Melanoma), and many others. Mutant Proteins themselves detected by Selected Reaction Monitoring (SRM) have been reported to be the most specific biomarkers for cancers because they can only come from an existing tumor [1-3].

ARC Journal of Clinical Case Reports

\subsection{Definitions of Cancer Biomarkers}

Organizations and publications vary in their definition of biomarker. In many areas of medicine, biomarkers are limited to proteins identifiable or measurable in the blood or urine. However, the term is often used to cover any molecular, biochemical, physiological, or anatomical property that can be quantified or measured.

The National Cancer Institute (NCI), in particular, defines biomarker as a: "A biological molecule found in blood, other body fluids, or tissues that is a sign of a normal or abnormal process, or of a condition or disease. A biomarker may be used to see how well the body responds to a treatment for a disease or condition. Also called molecular marker and signature molecule.

In cancer research and medicine, biomarkers are used in three primary ways:

- To help diagnose conditions, as in the case of identifying early stage cancers (Diagnostic)

- To forecast how aggressive a condition is, as in the case of determining a patient's ability to fare in the absence of treatment (Prognostic)

- To predict how well a patient will respond to treatment (Predictive) 


\section{Role OF BIOMARKERS IN CANCER RESEARCH AND MEDICINE}

\subsection{Uses of Biomarkers in Cancer Medicine}

\subsubsection{Risk Assessment}

Cancer biomarkers, particular those associated with genetic mutations or epigenetic alterations, often offer a quantitative way to determine when individuals are predisposed to particular types of cancers. Notable examples of potentially predictive cancer biomarkers include mutations on genes KRAS, p53, EGFR, erbB2 for colorectal, esophageal, liver, and pancreatic cancer; mutations of genes BRCA1 and BRCA2 for breast and ovarian cancer; abnormal methylation of tumor suppressor genes p16, CDKN2B, and p14ARF for brain cancer; hypermethylation of MYOD1, CDH1, and CDH13 for cervical cancer; and hypermethylation of $\mathrm{p} 16, \mathrm{p} 14$, and RB1, for oral cancer.

\subsubsection{Diagnosis}

Cancer biomarkers can also be useful in establishing a specific diagnosis. This is particularly the case when there is a need to determine whether tumors are of primary or metastatic origin. To make this distinction, researchers can screen the chromosomal alterations found on cells located in the primary tumor site against those found in the secondary site. If the alterations match, the secondary tumor can be identified as metastatic; whereas if the alterations differ, the secondary tumor can be identified as a distinct primary tumor.

\subsubsection{Prognosis and Treatment Predictions}

Another use of biomarkers in cancer medicine is for disease prognosis, which take place after an individual has been diagnosed with cancer. Here biomarkers can be useful in determining the aggressiveness of an identified cancer as well as its likelihood of responding to a given treatment. In part, this is because tumors exhibiting particular biomarkers may be responsive to treatments tied to that biomarker's expression or presence. Examples of such prognostic biomarkers include elevated levels of metallopeptidase inhibitor 1 (TIMP1), a marker associated with more aggressive forms of multiple myeloma,elevated estrogen receptor (ER) and/or progesterone receptor (PR) expression, markers associated with better overall survival in patients with breast cancer; HER2/neu gene amplification, a marker indicating a breast cancer will likely respond to trastuzumab treatment; a mutation in exon 11 of the proto-oncogene c-KIT, a marker indicating a gastrointestinal stromal tumor (GIST) will likely respond to imatinib treatment; and mutations in the tyrosine kinase domain of EGFR1, a marker indicating a patient's non-small-cell lung carcinoma (NSCLC) will likely respond to gefitinib or erlotinib treatment [4-7].

\subsubsection{Pharmacodynamics and Pharmacokinetics}

Cancer biomarkers can also be used to determine the most effective treatment regime for a particular person's cancer. Because of differences in each person's genetic makeup, some people metabolize or change the chemical structure of drugs differently. In some cases, decreased metabolism of certain drugs can create dangerous conditions in which high levels of the drug accumulate in the body. As such, drug dosing decisions in particular cancer treatments can benefit from screening for such biomarkers. An example is the gene encoding the enzyme thiopurine methyl-transferase (TPMPT). Individuals with mutations in the TPMT gene are unable to metabolize large amounts of the leukemia drug, mercaptopurine, which potentially causes a fatal drop in white blood count for such patients. Patients with TPMT mutations are thus recommended to be given a lower dose of mercaptopurine for safety considerations.

\subsubsection{Monitoring Treatment Response}

Cancer biomarkers have also shown utility in monitoring how well a treatment is working over time. Much research is going into this particular area, since successful biomarkers have the potential of providing significant cost reduction in patient care, as the current imagebased tests such as CT and MRI for monitoring tumor status are highly costly.

One notable biomarker garnering significant attention is the protein biomarker S100-beta in monitoring the response of malignant melanoma. In such melanomas, melanocytes, the cells that make pigment in our skin, produce the protein S100-beta in high concentrations dependent on the number of cancer cells. Response to treatment is thus associated with reduced levels of S100-beta in the blood of such individuals.

Similarly, additional laboratory research has shown that tumor cells undergoing apoptosis can release cellular components such as cytochrome c, nucleosomes, cleaved cytokeratin-18, and E-cadherin. Studies have found that these macromolecules and others can 
be found in circulation during cancer therapy, providing a potential source of clinical metrics for monitoring treatment.

\subsubsection{Recurrence}

Cancer biomarkers can also offer value in predicting or monitoring cancer recurrence. The Oncotype DX® breast cancer assay is one such test used to predict the likelihood of breast cancer recurrence. Thist test is intended for women with early-stage (Stage I or II), nodenegative, estrogen receptor-positive (ER+) invasive breast cancer who will be treated with hormone therapy. Oncotype DX looks at a panel of 21 genes in cells taken during tumor biopsy. The results of the test are given in the form of a recurrence score that indicates likelihood of recurrence at 10 years.

\subsection{Uses of Biomarkers in Cancer Research}

\subsubsection{Developing Drug Targets}

In addition to their use in cancer medicine, biomarkers are often used throughout the cancer drug discovery process. For instance, in the 1960s, researchers discovered the majority of patients with chronic myelogenous leukemia possessed a particular genetic abnormality on chromosomes 9 and 22 dubbed the Philadelphia chromosome. When these two chromosomes combine they create a cancer-causing gene known as BCR-ABL. In such patients, this gene acts as the principle initial point in all of the physiological manifestations of the leukemia. For many years, the BCR-ABL was simply used as a biomarker to stratify a certain subtype of leukemia. However, drug developers were eventually able to develop imatinib, a powerful drug that effectively inhibited this protein and significantly decreased production of cells containing the Philadelphia chromosome.

\subsubsection{Surrogate End points}

Another promising area of biomarker application is in the area of surrogate endpoints. In this application, biomarkers act as stand-ins for the effects of a drug on cancer progression and survival. Ideally, the use of validated biomarkers would prevent patients from having

Table1: types of Molecular cancer biomarkers to undergo tumor biopsies and lengthy clinical trials to determine if a new drug worked. In the current standard of care, the metric for determining a drug's effectiveness is to check if it has decreased cancer progression in humans and ultimately whether it prolongs survival. However, successful biomarker surrogates could save substantial time, effort, and money if failing drugs could be eliminated from the development pipeline before being brought to clinical trials.

Some ideal characteristics of surrogate endpoint biomarkers include:

- Biomarker should be involved in process that causes the cancer

- Changes in biomarker should correlate with changes in the disease

- Levels of biomarkers should be high enough that they can be measured easily and reliably

- Levels or presence of biomarker should readily distinguish between normal, cancerous, and precancerous tissue

- Effective treatment of the cancer should change the level of the biomarker

- Level of the biomarker should not change spontaneously or in response to other factors not related to the successful treatment of the cancer

Two areas in particular that is receiving attention as surrogate markers include circulating tumor cells (CTCs) and circulating miRNAs. Both these markers are associated with the number of tumor cells present in the blood, and as such, are hoped to provide a surrogate for tumor progression and metastasis. However, significant barriers to their adoption include the difficulty of enriching, identifying, and measuring CTC and miRNA levels in blood. New technologies and research are likely necessary for their translation into clinical care.

\section{TyPeS OF CANCER BIOMARKERS}

\section{Molecular cancer biomarkers}

\begin{tabular}{|l|l|}
\hline Tumor Type & Biomarker \\
\hline \multirow{2}{*}{ Breast } & ER/PR (estrogen receptor/progesteron receptor) \\
\cline { 2 - 2 } & HER-2/neu \\
\hline \multirow{3}{*}{ Colorectal } & EGFR \\
\cline { 2 - 2 } & KRAS \\
\cline { 2 - 2 } & UGT1A1 \\
\hline Gastric & HER-2/neu \\
\hline
\end{tabular}




\begin{tabular}{|l|l|}
\hline \multirow{4}{*}{ GIST } & c-KIT \\
\hline \multirow{5}{*}{ Leukemia/Lymphoma } & CD20 \\
\cline { 2 - 2 } & CD30 \\
\cline { 2 - 2 } & FIP1L1-PDGFRalpha \\
\cline { 2 - 2 } & PDGFR \\
\cline { 2 - 2 } & Philadelphia Chromosome (BCR/ABL) \\
\cline { 2 - 2 } & PML/RAR-alpha \\
\cline { 2 - 2 } & TPMT \\
\cline { 2 - 2 } & UGT1A1 \\
\hline Lung & EML4/ALK \\
\cline { 2 - 2 } & EGFR \\
\cline { 2 - 2 } & KRAS \\
\hline Melanoma & BRAF \\
\hline Pancreas & Elevated levels of leucine, isoleucine and valine \\
\hline
\end{tabular}

Other Examples of Biomarkers:

\section{REFERENCES}

- Tumor Suppressors Lost in Cancer

* Examples: BRCA1, BRCA2

- RNA

* Examples: mRNA, microRNA ${ }^{[60]}$

- Proteins found in body fluids or tissue.

* Examples: Prostate-specific antigen, and CA-125

\section{CONCLUSION}

Biomarkers factor into the diagnosis and treatment of almost every patient with cancer. When new pharmaceuticals are developed, they are required to pass high levels of scrutiny and be tested in carefully designed, randomized clinical trials prior to governmental approval. Unfortunately, similar requirements are not in place for biomarkers, although they too can significantly influence patient outcomes. Therefore, it is important for clinical, translational, and laboratory-based researchers to be acutely aware of the issues surrounding appropriate biomarker development, in order to facilitate entry of clinically useful biomarkers into the clinic, while avoiding the introduction of biomarkers that have not been sufficiently evaluated and therefore may be useless or even potentially detrimental to patient care.
[1] Calzone, Kathleen A. (2012). "Genetic Biomarkers of Cancer Risk". Seminars in Oncology Nursing. 28 (2): 122-128.

[2] Herceg, Zdenko; Hainaut, Pierre (2007-06$01)$. "Genetic and epigenetic alterations as biomarkers for cancer detection, diagnosis and prognosis". Molecular Oncology. 1 (1): 26-41.

[3] Li, Danni; Chan, Daniel W. (2014-0401). "Proteomic cancer biomarkers from discovery to approval: it's worth the effort". Expert Review of Proteomics. 11 (2): 135-136.

[4] Aizpurua-Olaizola, O.; Toraño, J. Sastre; Falcon-Perez, J.M.; Williams, C.; Reichardt, N.; Boons, G.-J. (2018). "Mass spectrometry for glycan biomarker discovery". TrAC Trends in Analytical Chemistry. 100: 7-14.

[5] Mishra, Alok; Verma, Mukesh (2010). "Cancer Biomarkers: Are We Ready for the Prime Time?". Cancers. 2 (1): 190-208.

[6] Rhea, Jeanne; Ross J. Molinaro (March 2011). "Cancer Biomarkers: Surviving the journey from bench to bedside". Medical Laboratory Observer. Retrieved 26 April 2013.

[7] Behne, Tara; Copur, M. Sitki (1 January 2012). "Biomarkers for Hepatocellular Carcinoma". International Journal of Hepatology. 2012: 1-7.

Citation: Nalini Kanta Sahoo, Madhusmita Sahu, A Short Review on Cancer Biomarkers. ARC Journal of Clinical Case Reports. 2019; 5(4): 6-9. doi:dx.doi.org/ 10.20431/2455-9806.0504002.

Copyright: () 2019 Authors. This is an open-access article distributed under the terms of the Creative Commons Attribution License, which permits unrestricted use, distribution, and reproduction in any medium, provided the original author and source are credited. 